\title{
Criterios sobre las tecnologías del aprendizaje y conocimiento (tac) en tiempo de pandemia covid-19
}

\section{Criteria on learning and knowledge technologies (tac) in times of pandemic covid-19}

DOI: $10.46932 / \mathrm{sfjdv} 2 \mathrm{n} 2-053$

Received in: january 1st, 2020

Accepted in: March 30th, 2020

\section{Gustavo Adolfo Santana Sardi}

Licenciado en Ciencias de la Educación Técnica mención Contabilidad Computarizada

Diplomado en Desarrollo de Estrategias Digitales de Aprendizaje

Magister en Gerencia Educativa

Analista del Centro de Promoción y Apoyo al Ingreso

Universidad Técnica de Manabí

E-mail: gusansar@hotmail.com

\section{Ricardo Larry Castro Coello}

Ingeniero Industrial

Magister en Gerencia Educativa

Magister en Calidad

Docente (Carrera Ingeniería Industrial)

Universidad Técnica de Manabí

E-mail: ricardo.castro@utm.edu.ec

\section{Jhimmy Andrés Gutiérrez Santana}

Licenciado en Ciencias de la Educación especialidad Psicología y Orientación Vocacional

Magister en Docencia e Investigación Educativa

Docente Agregado Tiempo Completo (Facultad de Ciencias Sociales y Humanísticas)

Universidad Técnica de Manabí

E-mail: jhimmyg@yahoo.com

Yita Maribel Briones Palacios

Licenciada en Ciencias de la Educación especialidad Psicología y Orientación Vocacional Magister en Orientación Educativa Vocacional y Profesional

Doctor Dentro del Programa de Doctorado en Educación

Docente Auxiliar 2 Tiempo Completo.

Universidad Técnica de Manabí

E-mail: yita.briones@utm.edu.ec

\section{Francisco Antonio Mawyin Cevallos}

Licenciado en Ciencias de la Educación especialidad Psicología y Orientación Vocacional Magister en Orientación Educativa Vocacional y Profesional

Doctor Dentro del Programa de Doctorado en Educación

Docente Auxiliar Tiempo Completo

Universidad Técnica de Manabí

E-mail: franciscomawyin2016@gmail.com 


\title{
RESUMEN
}

COVID-19 llegó al mundo para poner al revés los planes y fue tan fácil notar la falta de planificación en la aplicación de nuevas pedagogías y nuevas tecnologías, en todos los niveles educativos, puntualmente en las dirigidas a la enseñanza y el aprendizaje en línea conociendo a las herramientas pedagógicas y tecnológicas que permiten aprender y enseñar utilizando dispositivos conectados a Internet, superando la necesidad y limitación de reunir a docentes y alumnos en un mismo lugar físico para compartir un espacio común y también un mismo tiempo.

La era de la tecnología ha traído consigo cambios que son importantes en el marco educativo, no sólo por el impacto que tiene sobre las interacciones sociales, sino porque el mercado laboral también las arrastra a sus formas de convivencia y son parte clave de casi todos los procesos en estos tiempos de pandemia. En este artículo se mencionan algunos criterios donde ciertos autores y el banco mundial manifiestan su lado positivo y negativo respecto a las Tecnologías de aprendizajes y conocimiento en tiempos de pandemia.

Palabras claves: tecnologías de aprendizaje y conocimiento, pedagogías, docentes, planificación, COVID 19, Banco Mundial.

\begin{abstract}
COVID-19 came to the world to turn plans upside down and it was so easy to notice the lack of planning in the application of new pedagogies and new technologies, in all educational levels, punctually in those directed to teaching and learning online knowing the pedagogical and technological tools that allow learning and teaching using devices connected to the Internet, overcoming the need and limitation of gathering teachers and students in the same physical place to share a common space and also the same time.

The era of technology has brought with it changes that are important in the educational framework, not only because of the impact it has on social interactions, but also because the labor market also drags them into its forms of coexistence and they are a key part of almost all processes in these pandemic times.

This article mentions some criteria where certain authors and the world bank express their positive and negative side regarding learning and knowledge technologies in times of pandemic.
\end{abstract}

Keywords: learning and knowledge technologies, pedagogies, teachers, planning, COVID 19, World Bank.

\section{INTRODUCCION}

El acceso a una educación de calidad, como derecho fundamental de todas las personas, se enfrenta a un contexto de cambio paradigmático en el siglo XXI. El desarrollo que han alcanzado las Tecnologías de aprendizaje y la Comunicación (TAC) en la actualidad demanda de los sistemas educacionales una actualización constante de prácticas y contenidos que sean acordes a la nueva sociedad del aprendizaje. Pero a pesar de estos esfuerzos realizados durante los últimos años los sistemas educativos latinoamericanos, aún quedan problemas estructurales importantes que obstaculizan el logro de una educación de calidad con cobertura extendida en los países de la región, como son las crisis económicas, políticas, guerras y pandemias. 
A pesar de que la tecnología ha evolucionado con el pasar del tiempo y, desde que la informática comenzó a introducirse en la educación, muchos docentes no sólo no conocen mucho acerca de las nuevas tecnologías, sino que tampoco saben cómo manejar un computador o navegar en internet con un grado de conocimiento básico. Esto realmente se aprende mientras, otros docentes se capacitan para tener conocimiento aceptable en este campo y dan sus primeros pasos, y otros son además capaces de adquirir distintas formas y emplear las herramientas de información de modo creativo y de acuerdo a sus necesidades y las de sus alumnos.

Lo mismo sucede que, gracias a los esfuerzos que se continúan haciendo, todas las etapas de la educación incorporan estas tecnologías ya que constantemente aparecen otros nuevos procesos tecnológicos que modifican o reemplazan las anteriores pero se profundiza la idea cuando en el mundo existe un virus que les exige a los docentes su mayor esfuerzo para poder manejarlo. Afirma Sir Ken Robinson (2010) en una charla de TED que debido a los avances tecnológicos la educación actual está sufriendo una reforma, pero "las reformas ya no sirven, porque eso es simplemente mejorar un modelo que no funciona. Lo que necesitamos (...) no es una evolución sino una revolución”. Cabe mencionar en este apartado que el significado TED (Tecnología, Entretenimiento y Diseño) es un evento organizado de forma anual, celebrado originalmente en Monterrey, California en el año 1984. ... La idea central de las charlas TED es compartir ideas que apasionen, que motiven, que inspiren y transmitan creatividad.

En realidad, basar el aprendizaje sólo en el uso de herramientas concretas es una batalla perdida no sólo por lo apuntado, sino además porque son demasiadas y están en permanente cambio y renovación.

Como lo menciona Morín (2001), este considera que la gestión administrativa de la educación, debe generar un abordaje académico que permita el acercamiento al modo y forma en que las instituciones educativas deberían prepararse para abordar las nuevas realidades que convergen alrededor de pandemias, guerras y el mismo calentamiento global.

Precisamente, esta conjunción de tecnologías más metodología es lo que se ha dado en denominar TAC: tecnologías del aprendizaje y el conocimiento. Lozano (2011) las define así: "Las TAC tratan de orientar las tecnologías de la información y la comunicación (TIC) hacia unos usos más formativos, tanto para el estudiante como para el profesor, con el objetivo de aprender más y mejor. Se trata de incidir especialmente en la metodología, en los usos de la tecnología y no únicamente en asegurar el dominio de una serie de herramientas informáticas frente la pandemia del COVID 19. Se trata en definitiva de conocer y de explorar los posibles usos didácticos que las TIC tienen para el aprendizaje y la docencia. Es decir, las TAC van más allá de aprender a usar las TIC y apuestan por explorar estas herramientas tecnológicas al servicio del aprendizaje y de la adquisición de conocimiento." 
No se trata ciertamente de un nuevo modo de nombrar lo que ya conocemos (es decir las TIC y la metodología actual), sino que "en realidad lo que se plantea es cambiar el 'aprendizaje de la tecnología' por el 'aprendizaje con la tecnología', enfoque esté orientado totalmente al desarrollo de competencias metodológicas fundamentales como el aprender a aprender en un mundo de pandemia” (Lozano 2011).

Por el contrario, el solo hecho de poder presentar los contenidos de siempre por medios más atractivos para los estudiantes de hoy, más habituados a lo visual que al texto, ya es en sí mismo un valor porque puede facilitar el aprendizaje, pero se debe comprender que el papel que la informática debe desempeñar en la educación va mucho más allá de lo instrumental.

La actividad docente se encuentra desde hace tiempo, por lo menos en el país ecuatoriano en estado de insuficiencia. Mencionando de todas las razones culturales, políticas que han influido en este aspecto, los docentes tienen que reconocer que hay un aspecto en el que deben modificar cierta actitud si se quiere jerarquizar su profesión, con esto no tiene (o tal vez recién está naciendo) una tradición seria de perfeccionamiento permanente y de actualización.

\section{DESARROLLO}

La educación, por supuesto, no ha sido una excepción. A nivel global, nacional y local, el impacto es similar a otras actividades humanas y también asume formas particulares por las características específicas de las actividades docentes, de investigación y de extensión en el nivel educativo (Marinoni, Van’t Land, \& Jensen, 2020). El lento ritmo de cambio en las instituciones académicas a nivel mundial es lento, con enfoques de enseñanza basados en siglos de antigüedad, prejuicios institucionales arraigados y aulas pasadas de moda. Sin embargo, el COVID-19 se ha convertido en un catalizador para que las instituciones educativas de todo el mundo busquen soluciones innovadoras en un período de tiempo relativamente corto.

La decisión acerca de la clausura temporal de las instituciones educativas ha venido urgida por el principio de salvaguardar de la salud pública en un contexto en el que las grandes acumulaciones de personas generan, por la naturaleza de la pandemia, graves riesgos (UNESCO IESALC, 2020, p. 10).

El coronavirus está cambiando instantáneamente la forma en que se imparte la educación, ya que la educación y el hogar, ahora se convierten en el mismo lugar tras las necesarias regulaciones efectuadas. Según la UNESCO, más de 861.7 millones de esudiantes en 119 países se han visto afectados al tener que hacer frente a la pandemia global que nos ha sacudido este año. Millones de familias en EE.UU. se han tenido que unir al 1.7 millón de estudiantes que se encuentran enrolados en la educación en el hogar.

El contenido de los cursos y la preparación tecnológica recibida marcó profundamente las primeras actuaciones del docente en las aulas y derivó en una serie de propuestas desvinculadas del currículum y centradas en la herramienta informática (Álvarez, 2017). 
Para hacer una breve reseña las TIC, son más explotadas en las tres acciones de estudio, esto se debe a que este tipo de tecnología ha incidido en las funciones del sistema de educación y permitido la innovación en la transmisión de saberes nuevos(Lozano, 2011). Las diferentes instituciones educativas, poseedoras del conocimiento, han dejado prioridad en las fuentes del saber. Las TIC están ofreciendo a los estudiantes acceso a fuentes de conocimiento ilimitados, a herramientas multimedia que permiten ampliar estos conocimientos de información (Rodríguez, 2018). Mientras que las TAC, cuya finalidad es remodelar la metodología en cuanto al uso de la tecnología, pero no exclusivamente para asegurar el dominio de herramientas informáticas sino, más bien, para conocer, explorar los posibles usos didácticos que las TIC tienen en la enseñanza y la docencia para ponerlas al servicio del aprendizaje para la adquisición del conocimiento (Palacios, 2019).

Las Tecnologías del Aprendizaje y el Conocimiento (TAC) son parte fundamental de los ambientes virtuales de aprendizaje, están presentes en su desarrollo, en sus contenidos, en sus productos, con elementos didácticos que brindan a los estudiantes la oportunidad de obtener nuevos conocimientos, así como desarrollar sus habilidades y actitudes. De igual manera los facilitadores encuentran en ellas una infinidad de materiales que pueden incorporar en el diseño de recursos de enseñanza y en las actividades de aprendizajes de los estudiantes. Las TAC integran los equipos electrónicos y materiales digitales, diseñados y creados intencionalmente con propósitos educativos por especialistas en educación y tecnología, y se puede llamar, además, a ambientes de aprendizaje. (García, N. E \& Pérez, C. A. 2018).

Las TAC orientan las TIC hacia usos formativos, es decir, se integran en el proceso de EnseñanzaAprendizaje centrado en el estudiante. Además, potencializa habilidades para utilizar el conjunto de servicios, redes, software y dispositivos que ofrece la tecnología digital, con el fin de mejor la calidad de vida de los estudiantes. Con esta reorientación se muestra que las funciones deben estar relacionadas con sus estilos de aprendizaje, el uso de herramientas y el trabajo profesional. En otras palabras, los estudiantes necesitan compenetrarse tecnológicamente en el uso de herramientas de diversos ambientes para la resolución de problemas (Mineduc. 2013)

Al decir de Granados-Romero, López-Fernández, Avello Martíne, Luna-Álvarez, Luna-Álvarez \& Luna-Álvarez (2017), el objetivo de las TAC es remodelar la metodología del empleo de la tecnología, pero no sólo para asegurar el dominio de éstas sino, más bien, para conocer y usar las TIC como medios didácticos en el aprendizaje y adquisición de conocimientos.

En este sentido, educar en estos tiempos implica una formación compatible con nuevas formas de entretener, producir, aprender y trabajar, respetando los estilos de cada individuo y comunidad virtual. La generación Net es "atrevida, curiosa, desafiante, independiente, de fácil adaptabilidad, con autoestima alta y con orientación global” (Davila, 2006, p. 28) 
La óptima implementación de las TAC implica un cambio en la actuación del docente, se requiere sea innovador y capaz de transformar su propia superación (Moreno, 2015), para que desde su implementación esta tecnología deje de ser un simple discurso y se convierta en una acción práctica, en la que como agente promueva situaciones de desarrollo de capacidades para el aprendizaje; transformaciones en las que se adapten en el sistema educativo y representan la adopción de modelos pedagógicos fundamentados en nuevas metodologías con el fin de resolver el desencuentro existente entre las TIC y la educación. Siendo de esta forma que la adquisición y el manejo de cada uno de estas tecnologías en tiempos de COVID-19 sea la más efectiva al momento de realizar un modelo pedagógico hacia los estudiantes.

Ciertamente las TAC son una oportunidad para provocar el salto cualitativo en los sistemas educacionales en tiempos de pandemia siendo necesario para satisfacer las necesidades de la sociedad, que demanda cada vez más de profesionales capaces propulsar las constantes transformaciones que el propio desarrollo de la ciencia y la tecnología generan.

Con esto se pretende que los estudiantes desarrollen habilidades que les permitan aprender a aprender, generar nuevos conocimientos y experiencias y utilizar las herramientas digitales como un vehículo para innovar. Estas habilidades deben ir acompañadas de la responsabilidad en el uso de dichas herramientas.

Por otro lado, el rol del estudiante también se ve modificado por las herramientas tecnológicas, pues la tecnología implica una mayor motivación en el aprendizaje (Area, 2010; Pedró, 2011), así como un cambio de actitudes que enriquece el aprendizaje, y que contribuye a ganar confianza en uno mismo (Pedró, 2011). Pero estos recursos educativos necesitan ser dominados para la construcción del conocimiento, con lo cual el estudiante precisa de nuevas destrezas para afrontar esta realidad (Salinas, 2010).

Las Tecnologías del Aprendizaje y Conocimiento, para el Ciclo de Educación, incluye componentes interrelacionados para que los estudiantes desarrollen habilidades y destrezas que les permitan desarrollar sus competencias tecnológicas.

El primer componente, Habilidades tecnológicas, se enfoca en las habilidades que los estudiantes deben adquirir para personalizar su entorno de aprendizaje, recibir retroalimentación y transferir sus cocimientos con la aplicación de herramientas tecnológicas de manera eficiente.

El segundo componente, Recursos tecnológicos, promueve en los estudiantes el proponer proyectos de manera colaborativa a través de las distintas modalidades virtuales de empoderamiento, ser personas creativas, solidarias y socialmente activas garantizando con ello los aprendizajes inclusivos y significativos. 
El tercer componente, Producción e innovación tecnológica, impulsa el uso de manera responsable de la información o material digital, dentro del proceso enseñanza-aprendizaje para proponer respuestas innovadoras a situaciones de la vida cotidiana.

El área fue construida de forma participativa por especialistas y en años anteriores pasó por un período de validación tal como lo establece el Acuerdo Ministerial 91-2018 de fecha 9 de enero de 2018; en dicha validación participaron especialistas y docentes de todo el país.

Hablar de la actividad docente en el contexto de la educación implica reconocer transformaciones significativas en los modelos y didácticas de enseñanza-aprendizaje. En este punto, se debe hablar de la incorporación de las TAC no solo en el aula, ya que esto resultaría en un proceso técnico, sino en las metodologías de aprendizaje. La cámara digital, la filmadora, el ordenador, la grabadora de voz, el mismo teléfono celular, constituyen algunas de las herramientas con las que el docente y estudiante deben interactuar a lo largo de su formación profesional.

En el contexto educativo las Tecnologías del Aprendizaje y del Conocimiento, a diferencia de las TIC nos direccionan a un uso más pedagógico y formativo. Buscan llevar esta experiencia más allá del manejo informático o técnico a la implementación en pro de la adquisición de conocimiento y aprendizaje.

Para introducir de forma efectiva las TAC en nuestros sistemas educativos, Segura (2007) invita a tener en cuenta los siguientes aspectos:

- Actualización constante de conocimientos, habilidades, procesos y estrategias sobre los contenidos cognitivos y metacognitivos.

- Ver la enseñanza como un proceso complejo de continuo cambio y para toda la vida.

- La generación de entornos virtuales de aprendizaje.

- La transformación del rol docente y estudiante

En este sentido el estudiante también debe recordar que su rol implica el reconocimiento del uso de estas herramientas durante el tiempo de pandemia para enriquecer su aprendizaje, dejando a un lado su actitud de receptor y reproductor de contenidos y convirtiéndose en un usuario capaz de buscar, obtener, analizar, procesar, comunicar y transformar la información hasta convertirla en conocimiento para su aprendizaje.

Afirma Lozano (2011), “las TAC van más allá de aprender meramente a usar las TIC y apuestan por explorar estas herramientas tecnológicas al servicio del aprendizaje y de la adquisición de conocimiento.” Este tipo de formación resignifica las TIC y las pone al servicio del estudio y el trabajo, y permite una verdadera inclusión digital, que los docentes sabrán transmitir a los estudiantes. 
La introducción de las TAC en el ámbito educativo favorece en la adquisición del conocimiento, debido a que poseen herramientas que facilitan este proceso permitiéndole al estudiante tener un sin número de posibilidades para trabajar con independencia sintiéndose involucrado en su proceso educativo.

Según Banco Mundial (2020) la pandemia de COVID-19 (coronavirus) representa una amenaza para el avance de la educación en todo el mundo, ya que tiene dos impactos significativos:

- El cierre prácticamente universal de las instituciones educativas de todos los niveles.

- La recesión económica generada por las medidas de control de la pandemia.

Si no se realizan esfuerzos considerables para contrarrestar estos efectos, el cierre de instituciones educativas provocará pérdidas de aprendizaje, aumento de la deserción escolar y mayor desigualdad, que afecta a los hogares la cual agravará el daño debido a la reducción de la oferta y demanda educativa. Esos dos impactos tendrán, en conjunto, un costo a largo plazo sobre la calidad humana y el bienestar de los estudiantes.

Sin embargo, si los países reaccionan con rapidez para lograr que el aprendizaje no se interrumpa, pueden mitigar el daño e incluso transformar la recuperación en una nueva oportunidad.

Las respuestas en materia de políticas que se necesitan para alcanzar esa meta pueden resumirse en tres etapas superpuestas:

- Enfrentar la situación

- Gestionar la continuidad

- Mejorar y acelerar las actividades

Hoy en día la educación virtual en el Ecuador es la nueva modalidad que vive la estructura educativa general de los países, siendo esto un declive para los alrededores de las entidades formativas, por ello existe un impulso virtual emitido por las altas esferas gubernamentales en el no volver a la educación presencial para con esto tratar de reducir los contagios por el COVID-19, una presión directa para los estudiantes a través de las tecnologías que si bien es cierto no es paulatinamente desarrollada a nivel general ya que existen limitaciones de la misma, por lo cual es de suma importancia sacar a relucir estos temas que sin duda alguna forman parte del día a día de cada una de las personas.

Tomando en consideración lo planteado por Rubio (2015), Navarrete Mendieta y Mendienta (2018), Guachamín (2020), entre otros autores que hablan acerca de la tecnología en el entorno educativo frente a la pandemia, educación que hace que la nueva realidad mundial demanda cambiar la percepción de la educación tradicional, en donde muchos docentes y estudiantes al inicio de las clases virtuales presentaron dificultades en aspectos como temporalización y distribución de tareas, planificación de clases y desarrollo de las mismas, debido al proceso forzado en el uso de medios tecnológicos por el aislamiento generado por la pandemia, sin duda esta situación, será uno más de los factores que influirá en el 
aprendizaje de los educandos en todos sus niveles, el reto actual del sistema educativo en el país y el mundo se ubica en cómo enfrentar este momento, a través de medidas que permitan frenar brechas de desigualdad a nivel educativo.

Por otra parte, con respecto al portal de la Organización de Estados Iberoamericanos para la Educación, la Ciencia y la Cultura(2020) muestra a relucir los siguientes aspectos derivados de las tecnologías virtuales frente al covid-19: Para ello la capacitación y la comunicación efectiva con todos los miembros de la comunidad educativa permitirá la generación de competencias las cuales aportarán de manera significativa en el análisis y la generación de inferencias para asignar sentido a los mensajes en el ámbito virtual y los agrupamientos sociales en la cotidianidad de la web, en este caso a través de los entornos educativos virtuales, incidiendo en la identidad personal y pertenencia a cierta comunidad.

De acuerdo a las tecnologías aplicadas al conocimiento son aquellas que buscan ir más allá de enseñar a manejar un dispositivo sino más bien de interiorizar su funcionamiento para el logro del objetivo académico (Zambrano \& Balladares, (2017). No obstante, dentro de las TAC, se identifica a las plataformas virtuales de aprendizaje, las cuales aportan a la gestión de las actividades que se realizan dentro de la clase, para lo cual es importante definir su ámbito de trabajo (Prete \& Almenara, 2020).

El sistema de educación es una más de las víctimas de pandemia COVID-19, ante esta situación la educación debe continuar llevados a giros estratégicos para continuar con las clases.

El Ministerio de Educación del Ecuador MINEDUC, hizo lineamientos que orientaban cómo se deberían dar las clases virtuales y sobre todo se buscó enfatizar en la importancia de la planificación curricular, la cual asegura que el proceso de aprendizaje sea continuo, pertinente y en especial que sea dosificado. Todo este procedimiento permite que los docentes puedan organizar y dirigir de una manera adecuada cada una de las necesidades educativas que tienen los estudiantes. En una época de emergencia la planificación es necesaria y gracias a ella los docentes pueden generar rutinas y actividades coherentes, claras y mejorables de esa forma los beneficiados son los estudiantes. Es necesario que el docente busque generar en sus estudiantes dudas sobre cómo tener un autocuidado en esta pandemia, (Ministerio de Educación del Ecuador, 2020, p.10).

Las metodologías que se usan son aquellas que impelen a los estudiantes a ser los creadores de su propio aprendizaje, los cuales están enfocados en el respeto, la dignidad, la diversidad, es decir, un modelo integral, que este enfocado en incidir todos los valores humanos que permitan comprender el mundo y transformarlo mediante el diálogo y la participación.

Para obtener esos resultados, se propone herramientas creativas, las cuales busquen desafiar la capacidad del discente, de ese modo él puede fortalecer su creatividad, y así empezará a generar ideas innovadoras (Ministerio de Educación del Ecuador, 2020, p. 10). 
Con lo antes expuesto es necesario que el sistema educativo sufra un cambio radical hacia la transformación de la educación mediante la inclusión de herramientas tecnológicas para un aprendizaje significativo, pero debe ser un cambio de modelo que incorpore no solo lo que se crea adecuado desde un punto de vista pedagógico, sino por ejemplo integrar aristas que permitan incluso un desarrollo social sostenible, como lo indica Rojas-Londoño,(2019), es aquí cuando nace la imperante urgencia de incorporar al desarrollo social sostenible en un contexto dentro del campo educativo, mediante un enfoque pedagógico que tenga una visión completa de trabajo multi, inter y transdisciplinario, permitiendo implementar mediante acciones concretas la sostenibilidad empoderada en la sociedad del conocimiento y un modelo educativo eficaz y actualizado a las tendencias mundiales.

El aprendizaje significativo mediante la utilización de recursos tecnológicos que se dividen en tres componentes interdependientes, Tecnologías de la Información y Comunicación - TIC, las Tecnologías del Aprendizaje y del Conocimiento - TAC y complementadas para un engrane metodológico, necesitan incluso una nueva visión un nuevo paradigma que integre los componentes necesarios para una formación integral, es así que, Mármol-Jaramillo, Rojas-Londoño, (2020) señalan que, la socio formación se orienta a la solución de problemas abordando retos del desarrollo social sostenible, concibe la formación de personas integras comprometidas a satisfacer lo que demande la sociedad para mejorar las condiciones de vida sobre la base de un trabajo colaborativo y el pensamiento complejo en la consecución de un proyecto ético de vida; y, por tanto, en estrecha vinculación como actores principales los propios involucrados en la necesidad por resolver.

Esto ha provocado que los docentes incluyan dentro del proceso de enseñanza aprendizaje en estos tiempos, a raíz del COVID-19, una variedad de herramientas tecnológicas entre plataformas y recursos digitales, tanto lúdicos como técnicos, en cada uno de los aprendizajes didácticos, y tener que adaptar el currículo presencial a uno virtual para alcanzar un aprendizaje significativo y complementario a la realidad que vive la humanidad.

\section{CONCLUSIONES}

Las Instituciones de Educación Superior (IES) del mundo, y en particular las de América Latina, aún encaran dos momentos diferenciados, pero con características comunes. Por un lado, como se ha señalado, se ha intentado dar continuidad y concluir los procesos académicos abiertos antes o durante las primeras fases de esta crisis.

El propósito es contribuir a su desarrollo como personas creativas, solidarias y socialmente activas siendo desde el punto de vista educativo un reto para los docentes en tiempos de pandemia con el COVID19. 
Los criterios de evaluación son enunciados que tienen como función principal orienta a los docentes hacia los aspectos que se deben tener en cuenta al determinar el tipo y nivel de aprendizaje alcanzado por los estudiantes en cada uno de los momentos del proceso educativo, según las competencias establecidas en el currículo. Desde este punto de vista, puede decirse que las TAC funcionan como reguladores de las estrategias de aprendizaje-evaluación-enseñanza.

Esto demuestra como la tecnología incide mucho en la educación y podemos ver también el impacto que esta genera en la actualidad con la situación vivida por la pandemia COVID-19, dejando así muy claro la importancia que es la utilización de esta herramienta y más aún en momentos de crisis, una crisis que no solo nos afecta interior, sino que por el hecho de ser mundial se han tomado medidas que desmejoran los convenios de producción y desarrollo. 


\section{BIBLIOGRAFÍA}

Álvarez, R. E. (2017). Las TAC al servicio de la formación inicial de maestros en el área de Didáctica de la Lengua y la Literatura: herramientas, usos y problemática. Recuperado de https://www.researchgate. net/publication/321713163_Las_TAC_al_servicio_de_

la_formacion_inicial_de_maestros_en_el_area_de_Didactica_de_la_Lengua_y_la_Literatura_herramien tas_usos_y_problematica_LTK_at_the_service_of_initial_teacher_training_in_the_area

Area, M. (2010). El proceso de integración y uso pedagógico de las TIC en los centros educativos. Un estudio de casos. Revista Educación, 352, 77-97.

Banco mundial. (7 de mayo de 2020). Obtenido de covid19-pandemic-shocks-to-educationand-policyresponses: https://www.bancomundial.org/es/topic/education/publication/thecovid19-pandemic-shocksto-education-and-policy-responses

Davila, S. (2006). Generación Net: Visiones para su Educación Net Generation: Visions for their Education. Orbis, 24-48.

García, N. E \& Pérez, C. A. (2018). Creación de ambientes digitales de aprendizaje. Editorial digital UNID. Recuperado el 3 de mayo de 2019 de https://books.google. com.co/books?id=4d5PDwAAQBAJ\&printsec=frontcover\&source=gbs_ge_summa ry_r\&cad $=0 \# \mathrm{v}=$ onepage $\& \mathrm{q} \& \mathrm{f}=$ false

Granados-Romero, J., López-Fernández, R., Avello-Martínez, R., Luna-Álvarez, D., Luna-Álvarez, E., \& LunaÁlvarez, W. (2017). Las Tecnologías de la Información y las Comunicaciones, las del aprendizaje y del conocimiento y las tecnologías para el empoderamiento y la participación como instrumentos de apoyo al docente de la universidad del siglo XXI. Medisur, 12(1). Recuperado de http://www.medisur.sld.cu/index.php/ medisur/article/view/2751/1452

Guachamín, J. A. (25 de Mayo de 2020). Las dos caras de la educacion con el COVID - 19. Cienciamérica, 89-98. Obtenido de http://cienciamerica.uti.edu.ec/openjournal/index.php/uti/article/view/294/462

Lozano, R. "Las 'TIC/TAC': de las tecnologías de la información y comunicación a las tecnologías del aprendizaje y del conocimiento" 12/1/11. En http://www.thinkepi.net/las-tictac-de-las-tecnologias-de-lainformacion-y-comunicacion-a-las-tecnologias-delaprendizaje-y-del-conocimiento Último ingreso mayo de 2012.

Lozano, R. (2011). De las TIC a las TAC: tecnologías del aprendizaje y del conocimiento.Anuario ThinkEPI. https://recyt.fecyt.es/index.php/ThinkEPI/article/viewFile/30465/16032

Marinoni, Giorgio, Van't Land, Hilligje, \& Jensen, Trine. (2020). The Impact of COVID-19 on Higher Education Around the World IAU Global Survey Report. Retrieved from https://www.iauaiu.net/IMG/pdf/iau_covid19_and_he_survey_report_final_may_2020.pdf [ Links ]

Ministerio de Educación Nacional. (2013). Competencias TIC para el desarrollo profesional docente. Colección Sistema Nacional de Innovación Educativa con uso de Nuevas Tecnologías. 1a Edición - Obra Independiente ISBN: 978-958-750-762-1.

Ministerio de Educación del Ecuador. (2020, 12 de marzo). Comunicado Oficial Suspensión de las actividades académicas para los estudiantes para precautelar la salud de la comunidad educativa. 
Recuperado de https://educacion.gob.ec/comunicado-oficial-suspension-de-las-actividades-academicaspara-los-estudiantes-para-precautelar-la-salud-de-la-comunidad-educativa/.

Ministerio de Educación del Ecuador. (2020). Plan Educativo: aprendamos juntos en casa. Quito, Pichincha, Ecuador.

Moreno, M. (2015). Uso de las TIC en el aula. NUBEMIA. Recuperado d: https://www.nubemia.com/usode-lastic-en-el-aula

Morín, E. (2001). Los siete saberes necesarios para la educación del futuro. Nueva Visión. [ Links ]

Navarrete Mendieta, G., \& Mendienta , R. C. (Abril de 2018). LAS TIC Y LA EDUCACIÓN ECUATORIANA EN TIEMPOS DE. ales revista multidisciplinaria de investigación, 130-131.

Organización de Estados Ibeoamericanos. (2020). La ciencia y la tecnología como procesos sociales. Obtenido de https://www.oei.es/historico/salactsi/nunez05.htm

Palacios, J. (2019). Retos de la educación en la era del TIC, TAC, TEP.https://ensegundos.com.pa/2019/04/02/retos-de-la-educacion-en-la-era-del-tic-tac-tep/

Pedró, F. (2011). Tecnología y Escuela: lo que funciona y por qué. XXVI Semana Monográfica de la Educación. La Educación en la Sociedad Digital. Fundación Santillana. Recuperado de http://dide.minedu.gob.pe/handle/123456789/4870

Prete, A., \& Almenara, J. (2020). Las plataformas de formación virtual: algunas variables que determinan su utilización. Revista de Innovación Educativa, 11(2), 138-153.

Robinson, Ken (2010) "Bring on the learning revolution!". En http://blogs.educared.org/recomendacionestictac/2011/10/26/las-13-mejores conferenciasde-ted-sobreeducacion/. Último acceso junio de 2012

Rojas-Londoño, O. (2019). El desarrollo social sostenible: el eje de una nueva visión educativa. En L. G. Juárez-Hernández (Coord.), Memorias del Cuarto Congreso Internacional en Socioformación y Sociedad del Conocimiento (CIS-FOR-2019). México: CIFE.

Rodríguez, E. (2018). TIC, TAC y TEP: qué son, diferencias y similitudes.https://www.compartirpalabramaestra.org/actualidad/blog/tic-tac-y-tep-que-son-diferenciasy-similitudes

Rubio, A. Á. (2015). Jóvenes y generación 2020. revista de estudios de juventud, p.11

Salinas, J. (2010). Innovación docente y uso de las TIC en la enseñanza universitaria. Revista de Universidad y Sociedad del Conocimiento, 1(1). Recuperado de http://www.uoc.edu/rusc/dt/esp/salinas1104.pdf

Segura (2007). Las Tecnologías de la Comunicación y la Formación Docente. Guía de planificación. Montevideo. https://es.unesco.org/themes/tic-educacion 
UNESCO IESALC. (2020). Covid-19 y educación superior: de los efectos inmediatos al día después. Análisis de impactos, respuesta y recomendaciones. París, Francia: UNESCO. Recuperado de http://www.iesalc.unesco.org/wp-content/uploads/2020/04/COVID-19-060420-ES-2.pdf

Zambrano, F., \& Balladares, K. (2017). Sociedad del Conocimiento y las TEPs. INNOVA Research Journal, 2 (10), 169-177. 